\title{
The long-range non-additive three-body dispersion interactions for the rare gases, alkali and alkaline-earth atoms
}

\author{
Li-Yan Tang ${ }^{1}$, Zong-Chao Yan ${ }^{2,3}$, Ting-Yun Shi ${ }^{1}$, James F. Babb ${ }^{4}$ and J. Mitroy ${ }^{5}$ \\ 1 State Key Laboratory of Magnetic Resonance and Atomic and Molecular Physics, \\ Wuhan Institute of Physics and Mathematics, \\ Chinese Academy of Sciences, Wuhan 430071, P. R. China \\ ${ }^{2}$ Center for Cold Atom Physics, Chinese Academy of Sciences, Wuhan 430071, P. R. China \\ ${ }^{3}$ Department of Physics, University of New Brunswick, \\ Fredericton, New Brunswick, Canada E3B 5A3 \\ 4 ITAMP, Harvard-Smithsonian Center for Astrophysics, \\ Cambridge, Massachusetts 02138, USA and \\ 5 School of Engineering, Charles Darwin University, Darwin NT 0909, Australia
}

(Dated: May 22, 2018)

The long-range non-additive three-body dispersion interaction coefficients $Z_{111}, Z_{112}, Z_{113}$, and $Z_{122}$ are computed for many atomic combinations using standard expressions. The atoms considered include hydrogen, the rare gases, the alkali atoms (up to $\mathrm{Rb}$ ) and the alkalineearth atoms (up to Sr). The term $Z_{111}$, arising from three mutual dipole interactions is known as the Axilrod-Teller-Muto coefficient or the DDD (dipole-dipole-dipole) coefficient. Similarly, the terms $Z_{112}, Z_{113}$, and $Z_{122}$ arise from the mutual combinations of dipole (1), quadrupole (2), and octupole (3) interactions between atoms and they are sometimes known, respectively, as DDQ, DDO, and DQQ coefficients. Results for the four $Z$ coefficients are given for the homonuclear trimers, for the trimers involving two like-rare-gas atoms, and for the trimers with all combinations of the $\mathrm{H}, \mathrm{He}, \mathrm{Li}$ atoms. An exhaustive compilation of all coefficients between all possible atomic combinations is presented as supplementary data.

PACS numbers: 31.15.ac, 31.15.ap, 34.20.Cf

\section{INTRODUCTION}

The DDD (dipole-dipole-dipole) or Axilrod-Teller-Muto interaction [1] is a non-additive dispersion interaction that arises between three polarizable atoms or molecules. In the long-range potential energy function it enters at the order of the inverse ninth power of the characteristic separation of the three particles. It is studied in the physics and chemistry of gases, liquids, adsorption of atoms at surfaces, and materials science, and more recently for applications to ultracold atoms. In addition to the DDD interaction, there are terms arising from mutual interactions 
of higher-than-dipole induced multipoles [2, 3], such as the dipole-dipole-quadrupole (DDQ), the dipole-dipole-octupole (DDO), and the dipole-quadrupole-quadrupole (DQQ) interactions. Values of the coefficients are useful for creating models of potential energy surfaces and for comparison with more sophisticated methods that include exchange energies.

For a variety of reasons, there has been increased interest in the non-additive three-body dispersion coefficients. Some of the primary motivations come from studies of ultra-cold atoms. For example photo- and magneto-association techniques are being developed to form homonuclear and heteronuclear trimers. The collisions that govern such events typically occur at low energies where the importance of dispersion events are enhanced and there have been a number of studies that have investigated the impact of three-body non-additive interactions [4 7]. Another application area is in fine-tuning the interaction potentials of rare gases for the description of their bulk thermophysical properties such as the virial coefficients [8 12] and for computing properties of their solid forms [13]. There has also been interest in describing the structures of metal atoms embedded in rare gases [14-18]. Finally, the dispersion interactions give reference data that could be utilized to validate $a b$ initio calculations of the full three-body potential surfaces at large inter-nuclear separations [19-21].

The DDD coefficients are known very accurately for systems involving combinations of $\mathrm{H}$, He and Li through ab initio calculations [22 26], while the most accurate DDD values for the heavier rare gases are derived from pseudo-oscillator strength distributions (POSDs) using dipole polarizabilities and other experimental data [27 29]. Doran and Zucker [3] and Doran [30, 31] calculated higher order coefficients for the rare gases using Hartree-Fock methods. Their results were surpassed in accuracy by those of Thakkar et al [32]. Reasonably accurate values for the DDD coefficients were computed for the alkali-metal [33, 34] and for the alkaline-earth metal atoms [34]. An alternate approach to the determination of three-body dispersion coefficients is to use combination rules [35 38], which utilize estimates of other properties such as polarizabilities and two-body dispersion coefficients.

The present work represents the most comprehensive evaluation of three-body dispersion coefficients so far presented in the literature. Coefficients are computed using oscillator strength sum rules up to a combined inverse power of 15 in the interspecies separation distance are given. Extremely precise dispersion coefficients are given for all combinations of the $\mathrm{H}$, He and Li ground states since definitive results for these atoms can often serve as benchmarks to test methodologies used for more complicated systems. In addition, dispersion coefficients are given for all the threebody homonuclear trimers involving the rare gases, the alkali atoms up to $\mathrm{Rb}$, and the alkaline 
earth atoms up to Sr. Space requirements preclude the presentation of data for all possible trimers involving heteronuclear systems. Tabulated coefficients are only given for systems with two like rare gas atoms. The three-body dispersion coefficients for almost all other possible three-body systems are detailed in the supplementary data submitted with this paper.

\section{DEFINITIONS OF THE DISPERSION COEFFICIENTS}

\section{A. Expressions using oscillator strength sum rules}

The leading non-additive dipole-dipole-dipole dispersion interaction between three atoms (a, b, c) can be written as

$$
V_{111}^{\mathrm{abc}}\left(\mathbf{R}_{\mathrm{a}}, \mathbf{R}_{\mathrm{b}}, \mathbf{R}_{\mathrm{c}}\right)=Z_{111}^{\mathrm{abc}} W_{111}^{\mathrm{abc}}\left(\mathbf{R}_{\mathrm{a}}, \mathbf{R}_{\mathrm{b}}, \mathbf{R}_{\mathrm{c}}\right)
$$

where $Z_{111}^{\mathrm{abc}}$ is the three-body dispersion coefficient and the function $W_{111}^{\mathrm{abc}}\left(\mathbf{R}_{\mathrm{a}}, \mathbf{R}_{\mathrm{b}}, \mathbf{R}_{\mathrm{c}}\right)$ is detailed in $[1,[5]$, e.g.

$$
W_{111}^{\mathrm{abc}}\left(\mathbf{R}_{\mathrm{a}}, \mathbf{R}_{\mathrm{b}}, \mathbf{R}_{\mathrm{c}}\right)=\frac{1+3 \cos \left(\theta_{\mathrm{ab}}\right) \cos \left(\theta_{\mathrm{bc}}\right) \cos \left(\theta_{\mathrm{ca}}\right)}{R_{\mathrm{ab}}^{3} R_{\mathrm{bc}}^{3} R_{\mathrm{ca}}^{3}},
$$

where $\theta_{\mathrm{ab}}$ is the interior angle at $c$ due to atoms $a$ and $b, R_{\mathrm{ab}}$ is the distance between atoms $a$ and $b$, and

$$
\begin{aligned}
Z_{111}^{\mathrm{abc}} & =\frac{3}{2} \sum_{i j k} \frac{f_{\mathrm{a}, 0 i}^{(1)} f_{\mathrm{b}, 0 j}^{(1)} f_{\mathrm{c}, 0 k}^{(1)}}{\epsilon_{\mathrm{a}, 0 i} \epsilon_{\mathrm{b}, 0 j} \epsilon_{\mathrm{c}, 0 k}} \\
& \times \frac{\left(\epsilon_{\mathrm{a}, 0 i}+\epsilon_{\mathrm{b}, 0 j}+\epsilon_{\mathrm{c}, 0 k}\right)}{\left(\epsilon_{\mathrm{a}, 0 i}+\epsilon_{\mathrm{b}, 0 j}\right)\left(\epsilon_{\mathrm{a}, 0 i}+\epsilon_{\mathrm{c}, 0 k}\right)\left(\epsilon_{\mathrm{b}, 0 j}+\epsilon_{\mathrm{c}, 0 k}\right)} .
\end{aligned}
$$

The sums over $i, j$, and $k$ involve the dipole oscillator strengths $f_{0 i}^{(1)}$ and the energy difference $\epsilon_{0 i}$ from the ground state of each atom. There are alternate definitions of $Z_{111}$ where $Z_{111}$ is a factor of 3 smaller with the $W_{111}$ geometric factors having an extra multiplying factor of 3 [2, 31, 32].

More generally, the three-body potential for arbitrary multipole combinations can be written

$$
V_{\ell_{a} \ell_{b} \ell_{c}}^{\mathrm{abc}}\left(\mathbf{R}_{\mathrm{a}}, \mathbf{R}_{\mathrm{b}}, \mathbf{R}_{\mathrm{c}}\right)=Z_{\ell_{a} \ell_{b} \ell_{c}}^{\mathrm{abc}} W_{\ell_{a} \ell_{b} \ell_{c}}^{\mathrm{abc}}\left(\mathbf{R}_{\mathrm{a}}, \mathbf{R}_{\mathrm{b}}, \mathbf{R}_{\mathrm{c}}\right)
$$

where the general dispersion coefficient is written

$$
\begin{aligned}
Z_{\ell_{a} \ell_{b} \ell_{c}}^{a b c} & =\frac{3}{2} \sum_{i j k} \frac{f_{\mathrm{a}, 0 i}^{\left(\ell_{a}\right)} f_{\mathrm{b}, 0 j}^{\left(\ell_{b}\right)} f_{\mathrm{c}, 0 k}^{\left(\ell_{c}\right)}}{\epsilon_{\mathrm{a}, 0 i} \epsilon_{\mathrm{b}, 0 j} \epsilon_{\mathrm{c}, 0 k}} \\
& \times \frac{\left(\epsilon_{\mathrm{a}, 0 i}+\epsilon_{\mathrm{b}, 0 j}+\epsilon_{\mathrm{c}, 0 k}\right)}{\left(\epsilon_{\mathrm{a}, 0 i}+\epsilon_{\mathrm{b}, 0 j}\right)\left(\epsilon_{\mathrm{a}, 0 i}+\epsilon_{\mathrm{c}, 0 k}\right)\left(\epsilon_{\mathrm{b}, 0 j}+\epsilon_{\mathrm{c}, 0 k}\right)} .
\end{aligned}
$$


The $f_{0 i}^{(\ell)}$ refer to the multipole oscillator strength from the ground state of each atom according to the definitions in [26, 34], e.g.

$$
f_{0 i}^{(\ell)}=\frac{2}{(2 \ell+1)} \epsilon_{0 i}\left|\left\langle\Psi_{0} \| \sum_{j} r_{j}^{\ell} \mathbf{C}_{\ell}\left(\hat{\mathbf{r}}_{j}\right)|| \Psi_{i}\right\rangle\right|^{2} .
$$

The next term in the three-body dispersion series is the dipole-dipole-quadrupole (DDQ) contribution. In the case of a heteronuclear trimer there are three separate terms since the quadrupole excitation can be associated with any of the atoms, $a, b$, or $c$. One term is

$$
V_{112}^{\mathrm{abc}}\left(\mathbf{R}_{\mathrm{a}}, \mathbf{R}_{\mathrm{b}}, \mathbf{R}_{\mathrm{c}}\right)=Z_{112}^{\mathrm{abc}} W_{112}^{\mathrm{abc}}\left(\mathbf{R}_{\mathrm{a}}, \mathbf{R}_{\mathrm{b}}, \mathbf{R}_{\mathrm{c}}\right) .
$$

The $Z_{112}^{\text {abc }}$ coefficient is

$$
\begin{aligned}
Z_{112}^{\mathrm{abc}} & =\frac{3}{2} \sum_{i j k} \frac{f_{\mathrm{a}, 0 i}^{(1)} f_{\mathrm{b}, 0 j}^{(1)} f_{\mathrm{c}, 0 k}^{(2)}}{\epsilon_{\mathrm{a}, 0 i} \epsilon_{\mathrm{b}, 0 j} \epsilon_{\mathrm{c}, 0 k}} \\
& \times \frac{\left(\epsilon_{\mathrm{a}, 0 i}+\epsilon_{\mathrm{b}, 0 j}+\epsilon_{\mathrm{c}, 0 k}\right)}{\left(\epsilon_{\mathrm{a}, 0 i}+\epsilon_{\mathrm{b}, 0 j}\right)\left(\epsilon_{\mathrm{a}, 0 i}+\epsilon_{\mathrm{c}, 0 k}\right)\left(\epsilon_{\mathrm{b}, 0 j}+\epsilon_{\mathrm{c}, 0 k}\right)} .
\end{aligned}
$$

The spatial factor (dropping the arguments for compactness) for $W_{112}^{\text {abc }}$ is

$$
\begin{aligned}
W_{112}^{\mathrm{abc}} & =\frac{1}{16 R_{\mathrm{ab}}^{3} R_{\mathrm{bc}}^{4} R_{\mathrm{ca}}^{4}} \times\left[9 \cos \left(\theta_{\mathrm{ab}}\right)-25 \cos \left(3 \theta_{\mathrm{ab}}\right)\right. \\
& \left.+6 \cos \left(\theta_{\mathrm{bc}}-\theta_{\mathrm{ca}}\right)\left[3+5 \cos \left(2 \theta_{\mathrm{ab}}\right)\right]\right] .
\end{aligned}
$$

There is no need to give the $Z$ coefficients for the other combinations. The spatial factors for $W_{122}^{\text {abc }}$, $W_{113}^{\text {abc }}$ and $W_{222}^{\text {abc }}$ can be written [2, 5] as

$$
\begin{aligned}
W_{122}^{\mathrm{abc}} & =\frac{5}{64 R_{\mathrm{ab}}^{4} R_{\mathrm{bc}}^{5} R_{\mathrm{ca}}^{4}} \times\left[3 \cos \left(\theta_{\mathrm{bc}}\right)+15 \cos \left(3 \theta_{\mathrm{bc}}\right)\right. \\
& +20 \cos \left(\theta_{\mathrm{ca}}-\theta_{\mathrm{ab}}\right)\left[1-3 \cos \left(2 \theta_{\mathrm{bc}}\right)\right] \\
& \left.+70 \cos \left[2\left(\theta_{\mathrm{ca}}-\theta_{\mathrm{ab}}\right)\right] \cos \left(\theta_{\mathrm{bc}}\right)\right] \\
W_{113}^{\mathrm{abc}} & =\frac{5}{96 R_{\mathrm{ab}}^{3} R_{\mathrm{bc}}^{5} R_{\mathrm{ca}}^{5}} \times\left[9+8 \cos \left(2 \theta_{\mathrm{ab}}\right)-49 \cos \left(4 \theta_{\mathrm{ab}}\right)\right. \\
& \left.+6 \cos \left(\theta_{\mathrm{bc}}-\theta_{\mathrm{ca}}\right)\left[9 \cos \theta_{\mathrm{ab}}+7 \cos \left(3 \theta_{\mathrm{ab}}\right)\right]\right] \\
W_{222}^{\mathrm{abc}}= & \frac{5}{128 R_{\mathrm{ab}}^{5} R_{\mathrm{bc}}^{5} R_{\mathrm{ca}}^{5}} \times\left[490 \cos \left(2 \theta_{\mathrm{ab}}\right) \cos \left(2 \theta_{\mathrm{bc}}\right) \cos \left(2 \theta_{\mathrm{ca}}\right)\right. \\
- & 27+220 \cos \left(\theta_{\mathrm{ab}}\right) \cos \left(\theta_{\mathrm{bc}}\right) \cos \left(\theta_{\mathrm{ca}}\right) \\
+ & 175\left\{\cos \left[2\left(\theta_{\mathrm{bc}}-\theta_{\mathrm{ca}}\right)\right]+\cos \left[2\left(\theta_{\mathrm{ca}}-\theta_{\mathrm{ab}}\right)\right]\right. \\
+ & \left.\left.\cos \left[2\left(\theta_{\mathrm{ab}}-\theta_{\mathrm{bc}}\right)\right]\right\}\right]
\end{aligned}
$$


TABLE I: The dispersion constants (in a.u.) for homonuclear trimers consisting of either hydrogen, helium or lithium. All other dispersion coefficients are given by the symmetry conditions as described in the text. The numbers in parentheses are the computational uncertainties arising from incomplete convergence of the basis set. Values for hydrogen are correct to all quoted digits. The numbers in the square brackets denote powers of ten.

\begin{tabular}{lccccc}
\hline \hline System & $Z_{111}$ & $Z_{112}$ & $Z_{122}$ & $Z_{113}$ & $Z_{222}$ \\
\hline $\mathrm{H}$ & 21.642464510636 & 78.707166385334 & 289.37119056926 & 712.03297892387 & 1077.4737895329 \\
$\mathrm{He}$ & $1.47955860643(1)$ & $2.77280376179(1)$ & $5.21831397022(6)$ & $12.37878701399(5)$ & $9.8654795914(2)$ \\
$\mathrm{Li}$ & $1.70615(2)[5]$ & $1.78338(2)[6]$ & $1.99321(1)[7]$ & $5.12903(4)[7]$ & $2.463549(6)[8]$ \\
\hline \hline
\end{tabular}

Expressions for rearrangements of the different multipole excitations amongst the different atoms can be written down transparently. The expressions for the homonuclear trimers, i.e. $Z_{\ell_{1} \ell_{2} \ell_{3}}^{\text {aaa }}$ and $W_{\ell_{1} \ell_{2} \ell_{3}}^{\text {aaa }}$ do not require any modifications to the formulae listed above.

\section{B. Combination rules}

The present approach to the calculation of the dispersion parameters is based on explicit evaluation of oscillator strength sum rules. An alternative approach is to use integrals over the polarizabilities at imaginary frequencies [5, 26]. Approximate expressions that use existing calculations of polarizabilities and two-body dispersion parameters in combination rules have been derived [2, 35, 36]. One of these combination rules is known as the Midzuno-Kihara approximation [35, 36]. For a trimer consisting of three identical atoms, one has

$$
C_{9}=\frac{3}{4} \alpha_{d} C_{6}
$$

Other more complicated expressions exist for heteronuclear trimers [35, 36] and other combination rules have been developed [38]. Estimates using the Midzuno-Kihara approximation for $Z_{111}$ are given in some tables and abbreviated as MK.

\section{UNDERLYING DESCRIPTION OF ATOMIC STRUCTURES}

The evaluation of the three-body dispersion parameters involves the construction of tables of oscillator strengths to describe the excitation spectrum of all atoms considered. These oscillator strength distributions were constructed by a variety of means depending on the specifics of each 
atom. A short description is now given for each atom since the accuracy of the dispersion coefficients depends on the details of the underlying structure model.

\section{A. Hydrogen}

The hydrogen atom and its excitation spectrum was computed by diagonalising the hydrogen hamiltonian in a basis of Laguerre type orbitals (LTOs) [39, 40]. Such a basis has the property that it can be expanded to completeness without any linear dependence issues. Very accurate dispersion parameters can be obtained with relatively small basis sets.

\section{B. Hylleraas descriptions for $\mathrm{He}$ and $\mathrm{Li}$}

The helium atom wave function is expanded as a linear combination of Hylleraas functions. The resulting oscillator strength sum rules are capable of giving the dipole polarizability to an accuracy of 12 significant figures. The lithium atom wave function can also be represented as a linear combination of Hylleraas functions. The achievable accuracy for the Li ground state dipole polarizability is 5 significant figures. Details of the Hylleraas method and its application to He and Li can be found in Refs [17, 26].

\section{Pseudo-oscillator strength distributions for heavier rare gases}

The pseudo-oscillator strength distributions (POSDs) for the heavier rare gases, Ne, $\mathrm{Ar}, \mathrm{Kr}$ and Xe, come from two sources. The dipole distributions are taken from Kumar and Meath [27, 28], which were constructed by reference to polarizability data and other experimental data. The quadrupole and octupole pseudo-oscillator strengths are based on Hartree-Fock (HF) (or relativistic HF) single particle energies and radial expectation values. These were given tunings to reproduce calculations of higher multipole polarizabilities and homonuclear dispersion coefficients [41].

\section{Semi-empirical description of structure for alkali and alkaline-earth atoms}

The transition arrays for the alkali atoms are those which were used in calculations of the dispersion interactions between these atoms and the ground states of hydrogen and helium [42]. 
TABLE II: The three atom dispersion constants for combinations of $\mathrm{H}, \mathrm{He}$ and $\mathrm{Li}$ with two like atoms. The other dispersion constants are given by the symmetry relations $Z_{211}^{\mathrm{aab}}=Z_{121}^{\mathrm{aab}}, Z_{212}^{\mathrm{aab}}=Z_{122}^{\mathrm{aab}}$, and $Z_{311}^{\mathrm{aab}}=Z_{131}^{\mathrm{aab}}$ (in a.u.). The numbers in parentheses are the computational uncertainties arising from the finite basis set size.

\begin{tabular}{lcccc}
\hline \hline System & $Z_{111}^{\text {aab }}$ & $Z_{112}^{\text {aab }}$ & $Z_{121}^{\text {aab }}$ & $Z_{122}^{\text {aab }}$ \\
\hline H-H-He & $8.10224087465(2)$ & $14.6777067844(1)$ & $30.3021391145(1)$ & $55.1425238705(2)$ \\
H-H-Li & $275.987(3)$ & $4195.023(6)$ & $944.97(1)$ & $14670.29(2)$ \\
He-He-H & $3.26806489632(2)$ & $12.7469884056(1)$ & $5.99712674013(3)$ & $23.5605644280(1)$ \\
He-He-Li & $29.8259(6)$ & $504.658(1)$ & $53.228(1)$ & $907.467(2)$ \\
Li-Li-H & $6133.7(1)$ & $20621.1(2)$ & $72048.9(6)$ & $243884(2)$ \\
Li-Li-He & $1917.48(2)$ & $3394.56(4)$ & $22845.5(2)$ & $40500.9(4)$ \\
\hline System & $Z_{221}^{\text {aab }}$ & $Z_{113}^{\text {aab }}$ & $Z_{131}^{\text {aab }}$ & $Z_{222}^{\text {aab }}$ \\
\hline H-H-He & $115.1382084748(3)$ & $64.4548538033(2)$ & $277.5076627177(7)$ & $210.661799531(1)$ \\
H-H-Li & $3245.34(5)$ & $135490.15(9)$ & $8349.17259(1)$ & $51577.89(8)$ \\
He-He-H & $11.0328224804(1)$ & $118.9339509877(6)$ & $26.4993528199(1)$ & $43.6808551603(3)$ \\
He-He-Li & $95.057(2)$ & $17139.665(9)$ & $232.194(6)$ & $1633.531(4)$ \\
Li-Li-H & $966692(2)$ & $180949(2)$ & $214091(2)$ & $3312094(6)$ \\
Li-Li-He & $314438.8(7)$ & $14753.5(2)$ & $682925(7)$ & $558901(2)$ \\
\hline \hline
\end{tabular}

The transition arrays for the alkaline-earth atoms are those which were used in calculations of the dispersion interactions involving the alkali- and alkaline-earth atoms [34].

The wave function is partitioned into core and valence parts, where the core is described by a HF wave function. The ground and excited state pseudo-spectrum are computed by diagonalizing the fixed-core Hamiltonian of one and two electron functions written as a linear combination of LTOs. The HF core Hamiltonian is supplemented with a semi-empirical core polarization potential tuned to reproduce the energies of the low-lying spectrum. For all practical purposes the results are converged with respect to increasing the dimension of the orbital basis.

Excitations from the core are included in the calculations of the dispersion coefficients by the construction of pseudo-oscillator strength distributions based on HF energies and expectation values [34]. This approach to the determination of atomic structure is referred to as the configuration interaction plus core polarization (CICP) method in the remainder of this article. 


\section{NUMERICAL RESULTS}

\section{A. $\mathrm{H}, \mathrm{He}$ and $\mathrm{Li}$}

A complete set of dispersion coefficients for all combinations of the light systems involving $\mathrm{H}$, He and Li up to a total inverse power of $R^{15}$ are given in Tables I, II and III, The dispersion coefficients for these systems are given special prominence since they can be used to validate high precision quantum chemistry calculations of potential energy surfaces for these trimers. Table IV compares values of $Z_{111}$ with CICP results to give an accuracy benchmark for the CICP calculations.

Certain symmetry conditions apply when the trimer consists of three homonuclear atoms. One can write $Z_{121}^{\text {aaa }}=Z_{211}^{\text {aaa }}=Z_{112}^{\text {aaa }}, Z_{212}^{\text {aaa }}=Z_{221}^{\text {aaa }}=Z_{122}^{\text {aaa }}$, and $Z_{131}^{\text {aaa }}=Z_{311}^{\text {aaa }}=Z_{113}^{\text {aaa }}$. The results for the $\mathrm{H}$, He and Li homonuclear trimers are given in Table I. The dispersion coefficients for hydrogen are given to 14 digits. This does not represent the achievable precision. It is possible to compute the $Z_{\ell_{1} \ell_{2} \ell_{3}}$ to 30 significant digits by using quadruple precision arithmetic and a basis of 30 Laguerre functions. The $Z_{\ell_{1} \ell_{2} \ell_{3}}$ coefficients are in perfect agreement with those of Koga [25]. The Koga values use different conventions for relating the $Z_{\ell_{1} \ell_{2} \ell_{3}}$ to the geometric functions and are not quoted in Table $\mathrm{V}$

For helium, a precision of at least 10 significant digits was achieved, while for lithium six digits were tabulated with some uncertainty usually occurring in the sixth digit. There have been no previous high precision calculations of these coefficients except for $Z_{111}$ which were previously computed by Yan et al using the Hylleraas basis [26]. The present coefficients are in harmony with these earlier calculations.

The uncertainties in the dispersion parameters were estimated by an examination of the convergence for a series of successively larger calculations [17, 26]. Relativistic and finite mass corrections for these atoms can be expected to start to occur by the fourth to sixth digits. Therefore the precision achieved by the present non-relativistic calculations is sufficient for any practical calculation since finite-mass and relativistic corrections would need to be incorporated to further improve the overall level of accuracy.

The $Z_{111}$ coefficients for all H, He, Li combinations are given in Table IV. They are compared with the earlier Hylleraas calculations of Yan et al [26] and coefficients from from CICP type calculations [34]. The two sets of Hylleraas for trimers containing just $\mathrm{H}$ and $\mathrm{He}$ are generally in agreement when the quoted uncertainties are taken into consideration. However, the present dispersion coefficients for helium are about one order of magnitude more precise than the calculations 
TABLE III: The dispersion coefficients for the heteronuclear H-He-Li trimer (in a.u.). The numbers in parentheses are the computational uncertainties arising from incomplete convergence of the basis set.

\begin{tabular}{lccccc}
\hline \hline$Z_{\ell_{1} \ell_{2} \ell_{3}}$ & Values & $Z_{\ell_{1} \ell_{2} \ell_{3}}$ & Values & $Z_{\ell_{1} \ell_{2} \ell_{3}}$ & Values \\
\hline$Z_{111}$ & $89.833(1)$ & $Z_{112}$ & $1430.195(3)$ & $Z_{121}$ & $159.785(3)$ \\
$Z_{211}$ & $309.922(6)$ & $Z_{122}$ & $2557.314(4)$ & $Z_{212}$ & $5068.475(9)$ \\
$Z_{221}$ & $551.89(1)$ & $Z_{113}$ & $47174.62(3)$ & $Z_{131}$ & $695.90(1)$ \\
$Z_{311}$ & $2747.29(5)$ & $Z_{222}$ & $9081.12(2)$ & & \\
\hline \hline
\end{tabular}

TABLE IV: Comparison of the $Z_{111}$ (in a.u.) parameter for all combinations of the trimers formed by the $\mathrm{H}$, $\mathrm{He}$, and $\mathrm{Li}$ with some earlier calculations. The numbers in parentheses are the computational uncertainties arising from incomplete convergence of the basis set.

\begin{tabular}{lccc}
\hline \hline & & & Hylleraas \\
System & Present & Ref. [26] & Ref. [34] \\
\hline H-H-H & 21.64246451063597 & 21.642464510635 & 21.6425 \\
H-H-He & $8.10224087465(2)$ & $8.1022408743(2)$ & 8.103 \\
H-H-Li & $275.987(3)$ & $275.979(7)$ & 276.0 \\
He-He-He & $1.47955860643(1)$ & $1.4795586063(1)$ & 1.4798 \\
He-He-H & $3.26806489632(2)$ & $3.2680648961(1)$ & 3.269 \\
He-He-Li & $29.8259(6)$ & $29.824(5)$ & 29.83 \\
Li-Li-Li & $170615(2)$ & $170595(6)$ & 170873 \\
Li-Li-H & $6133.7(1)$ & $6133.5(5)$ & 6139 \\
Li-Li-He & $1917.48(2)$ & $1917.27(5)$ & 1919 \\
Li-He-H & $89.833(1)$ & $89.830(5)$ & 89.85 \\
\hline \hline
\end{tabular}

by Yan et al [26].

In a few instances involving the $\mathrm{Li}$ atom the present $Z_{111}$ values lie outside the error bars of the earlier Hylleraas calculation. The basis sets used in the Yan et al calculation were much smaller than those used herein. Agreement between the CICP and Hylleraas calculations is good, with no differences exceeding $0.2 \%$. The older $Z_{111}$ values of [2, 43] and [22] are not listed since they are not expected to have the precision of the present calculations.

The relative importance of the $\mathrm{Z}_{111}$ coefficient with respect to the two-body dispersion coefficients is easy to estimate and the $\mathrm{Li}-\mathrm{Li}-\mathrm{Li}$ trimer is used as an example. The two body $C_{8}$ and $C_{10}$ dispersion coefficients are $C_{8}=8.3429(1) \times 10^{4}$ a.u. and $C_{10}=7.3725(2) \times 10^{6}$ a.u. [17]. When three $\mathrm{Li}$ atoms form an equilateral triangle the spatial factor of Eq. (2) is $\frac{11}{8} R^{-9}$. At $R=30 a_{0}$ 
the ratios of $Z_{111} W_{111}$ to $C_{8} R^{-8}$ and $C_{10} R^{-10}$ are 0.28 and 0.95 respectively. The nonadditive three-body interaction should be included in high-precision analysis of photo-association spectra for homonuclear alkali-metal trimers. These ratios are 0.0096 and 0.1661 respectively for a helium trimer in an equilateral triangle configuration at $R=15 a_{0}$.

TABLE V: The three-body dispersion coefficients, $Z_{111}, Z_{112}, Z_{122}$ and $Z_{113}$ (in atomic units) for homonuclear trimers. The $f$-value distributions for $\mathrm{H}, \mathrm{He}$ and $\mathrm{Li}$ use Laguerre type orbitals $(\mathrm{H})$ or Hylleraas basis functions $(\mathrm{He}$ and $\mathrm{Li}$ ) to describe the ground and excited state spectra. Dispersion coefficients for the $\mathrm{H}$, He and Li trimers are given to additional significant digits in Table [ The heavier gas $f$-value distributions use pseudo-oscillator strength distributions [27, 28, 44], while those for the other atoms come from CICP calculations. Results in the Midzuno-Kihara (MK) approximation [35, 36] use the present oscillator strength distributions to compute the underlying $\alpha_{d}$ and $C_{6}$ needed as input. The numbers in the square brackets denote powers of ten.

\begin{tabular}{|c|c|c|c|c|c|c|c|}
\hline \multirow[t]{2}{*}{ Systems } & \multicolumn{3}{|c|}{$Z_{111}$} & \multicolumn{2}{|c|}{$Z_{112}$} & \multirow[t]{2}{*}{$Z_{122}$} & \multirow[t]{2}{*}{$Z_{113}$} \\
\hline & Present & Other & MK & Present & Other & & \\
\hline $\mathrm{He}$ & 1.4796 & $1.47956^{a}$ & 1.516 & 2.77280 & $2.77280^{b}$ & 5.2218 & 12.378 \\
\hline $\mathrm{Ne}$ & 11.95 & $12.37^{c}$ & 12.78 & 33.73 & $34.20^{d}$ & 95.21 & 189.7 \\
\hline $\mathrm{Ar}$ & 518.3 & $521.7^{c}$ & 534.4 & $2.543[3]$ & $2.554[3]^{a}$ & $1.251[4]$ & $2.535[4]$ \\
\hline $\mathrm{Kr}$ & $1.572[3]$ & $1.697[3]^{c}$ & $1.631[3]$ & $9.556[3]$ & $10.25[3]^{c}$ & $5.827[4]$ & $1.174[5]$ \\
\hline $\mathrm{Xe}$ & $5.573[3]$ & $6.246[3]^{c}$ & $5.822[3]$ & $4.582[4]$ & $5.163[4]^{c}$ & $3.778[5]$ & $7.298[5]$ \\
\hline $\mathrm{H}$ & 21.624 & $21.642^{b}$ & 21.93 & 78.707 & $78.72^{b}$ & 289.37 & 712.03 \\
\hline $\mathrm{Li}$ & $1.7062[5]$ & $1.701[5]^{d}$ & $1.717[5]$ & $1.786[6]$ & $1.750[6]^{d}$ & $1.997[7]$ & $5.138[7]$ \\
\hline $\mathrm{Na}$ & $1.892[5]$ & $1.906[5]^{d}$ & $1.908[5]$ & $2.544[6]$ & $2.353[6]^{g}$ & $3.553[7]$ & $7.844[7]$ \\
\hline $\mathrm{K}$ & $8.318[5]$ & $8.493[5]^{d}$ & $8.488[5]$ & $1.645[7]$ & $1.493[7]^{g}$ & $3.354[8]$ & $6.212[7]$ \\
\hline $\mathrm{Rb}$ & $1.063[6]$ & $1.097[5]^{d}$ & $1.097[6]$ & $2.455[8]$ & $2.230[8]^{g}$ & $5.678[8]$ & $9.704[8]$ \\
\hline $\mathrm{Be}$ & $5.973[3]$ & $6.135[3]^{e}$ & $6.023[3]$ & $5.335[5]$ & $5.535[4]^{e}$ & $4.865[5]$ & $7.420[5]$ \\
\hline $\mathrm{Mg}$ & $3.338[4]$ & $3.36[4]^{e}$ & $3.369[3]$ & $4.182[5]$ & $4.26[5]^{e}$ & $5.307[6]$ & $7.667[6]$ \\
\hline $\mathrm{Ca}$ & $2.557[5]$ & $3.25[5]^{e}$ & $2.626[5]$ & $5.131[6]$ & $5.34[6]^{e}$ & $1.034[8]$ & $1.125[8]$ \\
\hline $\mathrm{Sr}$ & $4.753[5]$ & $4.903[5]^{f}$ & $4.903[5]$ & $1.111[7]$ & & $2.601[8]$ & $2.983[8]$ \\
\hline
\end{tabular}

${ }^{a}$ Independent Hylleraas calculation [26]

${ }^{b}$ Pseudo oscillator strength distribution based on accurate oscillator strength sum rules [43] ${ }^{c}$ Many body perturbation theory (MBPT) [32]

${ }^{d}$ Model potential [33]

${ }^{e}$ Pseudo pscillator strength distributions (POSD). Value given is mean of the lower and upper limits. 29] ${ }^{f} \mathrm{MBPT}[31]$

${ }^{g}$ Asymptotic wave function [45] 


\section{B. Homonuclear trimers}

Dispersion coefficients for the homonuclear trimers are given in Table $\mathrm{V}$. The atoms presented are hydrogen, the rare gases, the alkali atoms, and the alkaline earth atoms. Values of $Z_{111}$ for most of these atoms have been published previously [22, 23, 26, 29, 31 34, 45-48].

The set of values for the rare gases heavier than He should be regarded as the current benchmark. The $Z_{111}$ coefficients use the pseudo oscillator strength distributions of Kumar and Meath [27, 28] and should be accurate to about $\pm 1 \%$. The best $a b$ initio calculation is the MBPT calculation [32] which should be regarded as superseding earlier calculations [3, 30, 31, 49, 50]. The MBPT calculation overestimates the dipole polarizabilities of $\mathrm{Kr}$ and Xe and this leads to the MBPT $Z_{111}$ values being too large for these atoms.

The present $Z_{112}$ values are slightly smaller than the MBPT values. The present values should be adopted as the recommended values for reasons outlined above since the most important multipole excitation is still the dipole and the pseudo oscillator strength distribution of Kumar and Meath is still the preferred value. The MBPT calculation [32] also gave values for $Z_{122}$ and $Z_{113}$. These are not tabulated for reasons of brevity since they repeat the same pattern seen for the $Z_{111}$ and $Z_{112}$ coefficients. The MBPT are about 5-10\% larger than the present values.

A detailed comparison of the CICP alkali atom $Z_{111}$ coefficients with those of a model potential calculation [33] listed in Table $\mathrm{V}$ has been made previously [34]. The CICP $Z_{111}$ coefficients were to be preferred since the model potential calculation [33] did not properly incorporate the contributions from the core. Two-body dispersion coefficients from the CICP calculations reproduce high quality relativistic many-body perturbation theory estimates to a high degree of precision [34, 51 [54].

For the alkali atoms there have also been estimates of the three-body dispersion coefficients based on a simple model potential tuned to give the correct binding energies for the lowest state in each angular momentum [45, 48]. The $Z_{112}$ results of Huang and Sun [45] are listed in TableV] The Huang and Sun $Z_{112}$ tend to be 5-10\% smaller than the present CICP values. Taking the Na-Na-Na trimer as a specific case, the Huang and Sun $Z_{112}$ of $2.353 \times 10^{6}$ a.u. value is $7 \%$ smaller than the present value. A similar situation also occurs for the two-body $C_{8}$ coefficient for the Na-Na dimer. The CICP calculations gives $1.159 \times 10^{5}$ a.u. [34, 55] while the Huang and Sun calculation gives $1.083 \times 10^{5}$ a.u. There are similar discrepancies with the Patil and Tang [48] tabulation. Patil and Tang give $2.35 \times 10^{6}$ a.u. for the Na-Ne-Ne $Z_{112}$, which is $7 \%$ smaller than the present value. The present CICP calculations expose the limitations of the structure models underlying the Huang 
and Sun [45] and Patil and Tang [48] tabulations. The Patil and Tang three-body coefficients, $Z_{111}$ and $Z_{112}$ were used as input in an attempt to make a global triatomic potential surface for three lithium atoms [5].

The present CICP $Z_{\ell_{1} \ell_{2} \ell_{3}}$ dispersion coefficients should be regarded as the reference values. For example, the $Z_{111}$ values from the Standard and Certain compilation [29] listed in Tables $\mathrm{V}$ are taken as the mean values of the lower and upper bounds. The oscillator strength distributions for some of the atoms in the Standard and Certain compilation rely on much older and smaller calculations.

\section{Impurity atoms embedded in the rare gases}

The three-body dispersion coefficients for the X-He-He systems given in Table VI, where X denotes $\mathrm{H}$, the alkali atoms up to $\mathrm{Rb}$ and the alkaline earth atoms up to Sr. The quadrupole excitation occurs on one of the He atoms for the $Z_{112}^{\mathrm{XHeHe}}=Z_{121}^{\mathrm{XHeHe}}$ coefficients. The quadrupole excitation is associated with the impurity $\mathrm{X}$ atom for the $Z_{211}^{\mathrm{XHeHe}}$ coefficient.

The three-body dispersion coefficients for the X-Ne-Ne and X-Ar-Ar systems are given in Table VII while those for the X-Kr-Kr and X-Xe-Xe systems are given in Table VIII. The quadrupole excitation is associated with the impurity $\mathrm{X}$ atom for the $Z_{211}^{\mathrm{XRgRg}}$ coefficient.

\section{Other systems}

The sheer number of possible heteronuclear three-body dispersion coefficients precludes their listing and discussion in the present manuscript. The three-body dispersion coefficients for every possible three-body trimer formed from the atoms studied in this paper ( $\mathrm{H}, \mathrm{He}, \mathrm{Ne}, \mathrm{Ar}, \mathrm{Kr}, \mathrm{Xe}$, $\mathrm{Li}, \mathrm{Na}, \mathrm{K}, \mathrm{Rb}, \mathrm{Be}, \mathrm{Mg}, \mathrm{Ca}, \mathrm{Sr})$ are given in the supplementary data published with this paper.

Previous compilations of the $Z_{111}^{a b c}$ three-body coefficients have been given for all possible alkaliatom trimers from $\mathrm{Li}$ to $\mathrm{Cs}\left[33\right.$, 34]. In addition, $Z_{111}^{a b c}$ coefficients for all possible alkaline-earth atoms from Be to Sr have also been published [34]. The supplementary data go well beyond these existing tabulations in that the higher-order $Z_{112}^{\mathrm{abc}}, Z_{113}^{\mathrm{abc}}$, and $Z_{122}^{\mathrm{abc}}$ are also given.

\section{E. The Midzuno-Kihara approximation}

The Midzuno-Kihara (MK) approximation [35, 36] for $Z_{111}$ has been given for all combinations in Tables V, VI VII and VIII. The coefficients in the MK approximation use the present oscillator 
TABLE VI: The three-body dispersion coefficients (in atomic units), for the X-He-He systems containing two helium atoms. The sources for the $f$-values distributions are the same as those in Table $\mathrm{V}$ The dispersion coefficients involving $\mathrm{H}$ and $\mathrm{Li}$ are given with additional significant digits in Table III Results in the Midzuno-Kihara (MK) approximation [35, 36] use the present oscillator strength distributions to compute the underlying $\alpha_{d}$ and $C_{6}$ needed as input. The numbers in the square brackets denote powers of ten.

\begin{tabular}{|c|c|c|c|c|c|c|c|c|}
\hline \multirow[t]{2}{*}{ Systems } & \multicolumn{2}{|c|}{$Z_{111}$} & \multirow[t]{2}{*}{$Z_{112}$} & \multirow[t]{2}{*}{$Z_{211}$} & \multirow[t]{2}{*}{$Z_{122}$} & \multirow[t]{2}{*}{$Z_{221}$} & \multirow[t]{2}{*}{$Z_{113}$} & \multirow[t]{2}{*}{$Z_{311}$} \\
\hline & Present & MK & & & & & & \\
\hline $\mathrm{Ne}$ & 2.955 & 3.067 & 5.561 & 8.335 & 10.51 & 15.69 & 24.86 & 46.96 \\
\hline $\mathrm{Ar}$ & 10.25 & 10.52 & 19.04 & 50.99 & 35.51 & 91.11 & 84.65 & 505.1 \\
\hline $\mathrm{Kr}$ & 14.62 & 15.05 & 27.09 & 90.66 & 50.37 & 168.6 & 120.3 & 1099 \\
\hline $\mathrm{Xe}$ & 21.74 & 22.46 & 40.15 & 183.2 & 74.35 & 339.4 & 177.9 & 2884 \\
\hline $\mathrm{H}$ & 3.269 & 3.324 & 5.999 & 12.75 & 11.04 & 23.57 & 26.52 & 118.9 \\
\hline $\mathrm{Li}$ & 29.83 & 30.13 & 53.25 & 505.1 & 95.11 & 908.5 & 232.6 & $1.716[4]$ \\
\hline $\mathrm{Na}$ & 33.52 & 34.30 & 59.99 & 624.8 & 107.5 & 1123 & 262.4 & $2.279[4]$ \\
\hline K & 50.73 & 52.82 & 90.97 & 1276 & 163.4 & 2287 & 398.1 & $6.014[4]$ \\
\hline $\mathrm{Rb}$ & 56.80 & 59.75 & 106.0 & 1538 & 183.6 & 2753 & 447.0 & $7.676[4]$ \\
\hline $\mathrm{Be}$ & 16.54 & 16.77 & 29.90 & 176.3 & 54.13 & 321.2 & 131.3 & 2749 \\
\hline $\mathrm{Mg}$ & 27.05 & 27.56 & 48.78 & 392.2 & 88.07 & 710.1 & 213.9 & 8249 \\
\hline $\mathrm{Ca}$ & 46.34 & 47.87 & 83.42 & 1008 & 150.4 & 1814 & 365.7 & $3.047[4]$ \\
\hline $\mathrm{Sr}$ & 55.97 & 58.23 & 100.8 & 1354 & 181.8 & 2433 & 442.0 & $4.629[4]$ \\
\hline
\end{tabular}

strength distributions to compute the underlying $\alpha_{d}$ and $C_{6}$ needed as input. In every instance the MK results are larger by an amount between 1-4\% than those of the explicit calculation.

\section{CONCLUSIONS}

A large scale investigation of the three-body dispersion coefficients for a set of 14 atoms has been completed. The results presented for the set of small systems, namely $\mathrm{H}$, He and Li should be regarded as the existing calculational standard.

The current set of values should also be adopted as the reference standard to be used for heavier systems. Even though more approximate methods of solving the Schrödinger equation are adopted, the semi-empirical Hamiltonian adopted for the alkali atoms and the alkaline earth atoms reduces the computational complexity to such an extent that uncertainties in the solution of the resulting Schrödinger equation are largely eliminated. When comparisons of polarizabilities and two-body 
TABLE VII: The three-body dispersion coefficients (in atomic units), for systems containing two neon atoms and two argon atoms. The sources for the $f$-values distributions are the same as those in Table $\mathrm{V}$ Results in the Midzuno-Kihara (MK) approximation [35, 36] use the present oscillator strength distributions to compute the underlying $\alpha_{d}$ and $C_{6}$ needed as input. The numbers in the square brackets denote powers of ten.

\begin{tabular}{|c|c|c|c|c|c|c|c|c|}
\hline \multirow[t]{2}{*}{ Systems } & \multicolumn{2}{|c|}{$Z_{111}$} & \multirow[t]{2}{*}{$Z_{112}$} & \multirow[t]{2}{*}{$Z_{211}$} & \multirow[t]{2}{*}{$Z_{122}$} & \multirow[t]{2}{*}{$Z_{221}$} & \multirow[t]{2}{*}{$Z_{113}$} & \multirow[t]{2}{*}{$Z_{311}$} \\
\hline & Present & MK & & & & & & \\
\hline \multicolumn{9}{|c|}{$\mathrm{X}-\mathrm{Ne}-\mathrm{Ne}$} \\
\hline $\mathrm{H}$ & 12.64 & 13.01 & 35.61 & 49.82 & 100.3 & 140.4 & 200.5 & 467.3 \\
\hline $\mathrm{Li}$ & 111.9 & 113.6 & 315.2 & 1907 & 887.9 & 5370 & 1768 & $6.502[4]$ \\
\hline $\mathrm{Na}$ & 126.2 & 130.2 & 355.6 & 2567 & 1002 & 6638 & 1994 & $8.629[4]$ \\
\hline K & 191.6 & 200.9 & 539.9 & 4803 & 1521 & $1.353[4]$ & 3028 & $2.268[5]$ \\
\hline $\mathrm{Rb}$ & 215.1 & 228.0 & 606.0 & 5785 & 1707 & $1.630[4]$ & 3399 & $2.893[5]$ \\
\hline $\mathrm{Be}$ & 62.79 & 64.37 & 176.9 & 674.8 & 498.3 & 1901 & 994.2 & $1.060[4]$ \\
\hline $\mathrm{Mg}$ & 102.5 & 105.6 & 288.8 & 1491 & 813.5 & 4199 & 1622 & $3.156[4]$ \\
\hline $\mathrm{Ca}$ & 175.6 & 183.1 & 494.6 & 175.6 & 1393 & $1.073[4]$ & 2776 & $1.157[5]$ \\
\hline $\mathrm{Sr}$ & 212.2 & 223.0 & 598.0 & 5111 & 1685 & $1.440[4]$ & 3356 & $1.754[5]$ \\
\hline \multicolumn{9}{|c|}{ X-Ar-Ar } \\
\hline $\mathrm{H}$ & 174.0 & 177.9 & 846.6 & 658.1 & 4127 & 3217 & 8470 & 6056 \\
\hline $\mathrm{Li}$ & 1818 & 1840 & 8704 & $2.955[4]$ & $4.170[4]$ & $1.420[5]$ & $8.804[4]$ & $9.836[5]$ \\
\hline $\mathrm{Na}$ & 2022 & 2068 & 9690 & $3.675[4]$ & $4.647[4]$ & $1.766[5]$ & $9.793[4]$ & $1.314[6]$ \\
\hline K & 3041 & 3169 & $1.459[4]$ & $7.636[4]$ & $7.006[4]$ & $3.662[5]$ & $1.474[5]$ & $3.532[6]$ \\
\hline $\mathrm{Rb}$ & 3378 & 3557 & $1.622[4]$ & $9.220[4]$ & $7.798[4]$ & $4.208[5]$ & $1.638[5]$ & $4.525[6]$ \\
\hline $\mathrm{Be}$ & 945.6 & 962.3 & 4557 & 9714 & $2.198[4]$ & $4.704[4]$ & $4.585[4]$ & $1.476[5]$ \\
\hline $\mathrm{Mg}$ & 1568 & 1600 & 7544 & $2.220[4]$ & $3.633[4]$ & $1.071[5]$ & $7.599[4]$ & $4.548[5]$ \\
\hline $\mathrm{Ca}$ & 2719 & 2812 & $1.307[4]$ & $5.890[4]$ & $6.290[4]$ & $2.832[5]$ & $1.318[5]$ & $1.732[6]$ \\
\hline $\mathrm{Sr}$ & 3279 & 3415 & $1.577[4]$ & $7.970[4]$ & $7.590[4]$ & $3.829[5]$ & $1.590[5]$ & $2.655[6]$ \\
\hline
\end{tabular}

dispersion coefficients have been made the CICP approach has typically been within $1 \%$ of the most sophisticated approaches to atomic structure available [34, 54].

Besides an improvement in accuracy, the scope of the present work exceeds that of any previous work by at least an order of magnitude. For example, Huang and Sun [45], and Patil and Tang [48]. presented extensive results for the alkali atoms. However, the present calculations also encompass the rare gases and the alkaline-earth atoms. 
TABLE VIII: The three-body dispersion coefficients (in atomic units), for systems containing two krypton or two xenon atoms. The sources for the $f$-values distributions are the same as those in Table $\mathrm{V}$ Coefficients in the Midzuno-Kihara (MK) approximation [35, 36] use the present oscillator strength distributions to compute the underlying $\alpha_{d}$ and $C_{6}$ needed as input. All values are in atomic units. The numbers in the square brackets denote powers of ten.

\begin{tabular}{|c|c|c|c|c|c|c|c|c|}
\hline \multirow[t]{2}{*}{ Systems } & \multicolumn{2}{|c|}{$Z_{111}$} & \multirow[t]{2}{*}{$Z_{112}$} & \multirow[t]{2}{*}{$Z_{211}$} & \multirow[t]{2}{*}{$Z_{122}$} & \multirow[t]{2}{*}{$Z_{221}$} & \multirow[t]{2}{*}{$Z_{113}$} & \multirow[t]{2}{*}{$Z_{311}$} \\
\hline & Present & MK & & & & & & \\
\hline \multicolumn{9}{|c|}{$\mathrm{X}-\mathrm{Kr}-\mathrm{Kr}$} \\
\hline $\mathrm{H}$ & 370.1 & 380 & 2485 & 1385 & $1.352[4]$ & 8409 & $2.760[4]$ & $1.268[4]$ \\
\hline $\mathrm{Li}$ & 4081 & 4136 & $2.407[4]$ & $6.612[4]$ & $1.420[5]$ & $3.870[5]$ & $3.044[5]$ & $2.151[6]$ \\
\hline $\mathrm{Na}$ & 4521 & 4628 & $2.671[4]$ & $8.216[4]$ & $1.579[5]$ & $4.819[5]$ & $3.372[5]$ & $2.881[6]$ \\
\hline K & 6795 & 7.086 & $4.017[4]$ & $1.700[5]$ & $2.377[5]$ & $1.006[6]$ & $5.070[5]$ & $7.800[6]$ \\
\hline $\mathrm{Rb}$ & 7526 & 7934 & $4.454[4]$ & $2.055[5]$ & $2.639[5]$ & $1.215[6]$ & $5.616[5]$ & $1.000[7]$ \\
\hline $\mathrm{Be}$ & 2066 & 2109 & $1.232[4]$ & $2.092[4]$ & $7.354[4]$ & $1.256[5]$ & $1.539[5]$ & $3.147[5]$ \\
\hline $\mathrm{Mg}$ & 3445 & 3523 & $2.049[4]$ & $4.831[4]$ & $1.220[5]$ & $2.885[5]$ & $2.567[5]$ & $9.796[5]$ \\
\hline $\mathrm{Ca}$ & 6015 & 6229 & $3.569[4]$ & $1.299[5]$ & $2.120[5]$ & $7.714[5]$ & $4.484[5]$ & $3.774[6]$ \\
\hline $\mathrm{Sr}$ & 7253 & 7563 & $4.304[4]$ & $1.763[5]$ & $2.557[5]$ & $1.046[6]$ & $5.407[5]$ & $5.807[6]$ \\
\hline \multicolumn{9}{|c|}{$\mathrm{X}-\mathrm{Xe}-\mathrm{Xe}$} \\
\hline $\mathrm{H}$ & 871.4 & 899.3 & 7139 & 3217 & $5.862[4]$ & $2.650[4]$ & $1.139[5]$ & $2.930[4]$ \\
\hline $\mathrm{Li}$ & $1.034[4]$ & $1.049[4]$ & $8.232[4]$ & $1.610[5]$ & $6.558[5]$ & $1.294[6]$ & $1.326[6]$ & $5.255[6]$ \\
\hline $\mathrm{Na}$ & $1.140[4]$ & $1.168[4]$ & $9.092[4]$ & $2.013[5]$ & $7.257[5]$ & $1.616[6]$ & $1.464[6]$ & $7.059[6]$ \\
\hline K & $1.713[4]$ & $1.787[4]$ & $1.366[5]$ & $4.528[5]$ & $1.091[6]$ & $3.405[6]$ & $2.200[6]$ & $4.258[7]$ \\
\hline $\mathrm{Rb}$ & $1.891[4]$ & $1.994[4]$ & $1.510[5]$ & $5.157[5]$ & $1.208[6]$ & $4.120[6]$ & $2.430[6]$ & $2.483[7]$ \\
\hline $\mathrm{Be}$ & 5042 & 5162 & $4.072[4]$ & $5.009[4]$ & $3.293[5]$ & $4.076[5]$ & $6.531[5]$ & $7.439[5]$ \\
\hline $\mathrm{Mg}$ & 8476 & 8690 & $6.824[4]$ & $1.172[5]$ & $5.500[5]$ & $9.489[5]$ & $1.095[6]$ & $2.345[6]$ \\
\hline $\mathrm{Ca}$ & $1.494[4]$ & $1.549[4]$ & $1.199[4]$ & $3.210[5]$ & $9.625[5]$ & $2.580[6]$ & $1.962[6]$ & $9.177[6]$ \\
\hline $\mathrm{Sr}$ & $1.802[4]$ & $1.881[4]$ & $1.445[5]$ & $4.377[5]$ & $1.161[6]$ & $3.512[6]$ & $2.323[6]$ & $1.419[7]$ \\
\hline
\end{tabular}

\section{Acknowledgments}

This work was supported by NNSF of China under Grant Nos. 11104323 and 11034009, and by the National Basic Research Program of China under Grant No. 2012CB821305. Z.-C.Y. was supported by NSERC of Canada and by the computing facilities of ACEnet, SHARCnet, WestGrid, and in part by the CAS/SAFEA International Partnership Program for Creative Research Teams. J. M. would like to thank the Wuhan Institute of Physics and Mathematics for its hospitality 
during his visits. The work of J.M was supported in part by the Australian Research Council Discovery Project DP-1092620. ITAMP is partially supported by a grant from the US NSF to Harvard University and the Smithsonian Astrophysical Observatory.

[1] B. M. Axilrod and E. Teller, J. Chem. Phys. 11, 299 (1943).

[2] R. J. Bell, J. Phys. B 3, 751 (1970).

[3] M. B. Doran and I. J. Zucker, J. Phys. C 4, 307 (1971).

[4] P. Soldán, M. T. Cvitaš, and J. M. Hutson, Phys. Rev. A 67, 054702 (2003).

[5] M. T. Cvitaš, P. Soldán, and J. M. Hutson, Mol. Phys. 104, 23 (2006).

[6] M. T. Cvitaš, P. Soldán, J. M. Hutson, P. Honvault, and J.-M. Launay, J. Chem. Phys. 127, 074302 (2007).

[7] G. Quéméner, J.-M. Launay, and P. Honvault, Phys. Rev. A 75, 050701 (2007).

[8] M. A. van der Hoef and P. A. Madden, J. Chem. Phys. 111, 1520 (1999).

[9] N. Jakse, J. M. Bomont, and J. L. Bretonnet, J. Chem. Phys. 116, 8504 (2002).

[10] A. E. Nasrabad, R. Laghaei, and U. K. Deiters, J. Chem. Phys. 121, 6423 (2004).

[11] L. Wang and R. J. Sadus, Phys. Rev. E 74, 021202 (2006).

[12] B. Jäger, R. Hellmann, E. Bich, and E. Vogel, J. Chem. Phys. 135, 084308 (2011).

[13] V. F. Lotrich and K. Szalewicz, Phys. Rev. Lett. 79, 1301 (1997).

[14] W. E. Baylis, J. Phys. B 10, L477 (1977).

[15] K. Niemax, J. Phys. B 13, 1791 (1980).

[16] X. W. Sheng, P. Li, and K. T. Tang, J. Chem. Phys. 130, 174310 (2009).

[17] L.-Y. Tang, Z.-C. Yan, T.-Y. Shi, and J. F. Babb, Phys. Rev. A 79, 062712 (2009).

[18] G. P. Yin, P. Li, and K. T. Tang, J. Chem. Phys. 132, 074303 (2010).

[19] A. W. Hauser, C. Callegari, P. Soldán, and W. E. Ernst, J. Chem. Phys. 129, 044307 (2008).

[20] P. Soldán, J. Chem. Phys. 132, 234308 (2010).

[21] P. Soldán, Phys. Rev. A 82, 034701 (2010).

[22] G. M. Stacey and A. Dalgarno, J. Chem. Phys. 48, 2515 (1968).

[23] R. F. Stewart, J. Phys. B 6, 2213 (1973).

[24] T. Koga, J. Chem. Phys. 84, 1636 (1986).

[25] T. Koga, J. Chem. Phys 90, 605 (1989).

[26] Z. C. Yan, J. F. Babb, A. Dalgarno, and G. W. F. Drake, Phys. Rev. A 54, 2824 (1996).

[27] A. Kumar and W. J. Meath, Mol. Phys. 54, 823 (1985).

[28] A. Kumar and W. J. Meath, Can. J. Chem. 63, 1616 (1985).

[29] J. M. Standard and P. R. Certain, J. Chem. Phys. 83, 3002 (1985).

[30] M. B. Doran, J. Phys. B 5, L151 (1972). 
[31] M. B. Doran, J. Phys. B 7, 558 (1974).

[32] A. J. Thakkar, H. Hettema, and P. E. S. Wormer, J. Chem. Phys. 97, 3252 (1992).

[33] M. Marinescu and A. F. Starace, Phys. Rev. A 55, 2067 (1997).

[34] J. Mitroy and M. W. J. Bromley, Phys. Rev. A 68, 052714 (2003).

[35] Y. Midzuno and T. Kihara, J. Phys. Soc. Jpn 11, 1045 (1956).

[36] T. Kihara, Adv. Chem. Phys. 1, 267 (1958).

[37] K. T. Tang, Phys. Rev. 177, 108 (1969).

[38] M. Diaz Peña, C. Pando, and J. A. R. Renuncio, J. Chem. Phys. 73, 1750 (1980).

[39] Z. C. Yan and A. Dalgarno, Mol. Phys. 96, 863 (1999).

[40] J. Mitroy and M. W. J. Bromley, Phys. Rev. A 71, 032709 (2005).

[41] J. Mitroy and J. Y. Zhang, Phys. Rev. A 76, 032706 (2007).

[42] J. Y. Zhang and J. Mitroy, Phys. Rev. A 76, 022705 (2007).

[43] R. J. Bell and J. Drake, J. Phys. B 3, L101 (1970).

[44] J. Mitroy and M. W. J. Bromley, Phys. Rev. Lett. 98, 063401 (2007).

[45] S. Huang and Q. Sun, J. Chem. Phys. 134, 144110 (2011).

[46] P. W. Langhoff and M. Karplus, J. Chem. Phys. 53, 233 (1970).

[47] D. J. Margoliash and W. J. Meath, J. Chem. Phys. 68, 1426 (1978).

[48] S. H. Patil and K. T. Tang, J. Chem. Phys. 106, 2298 (1997).

[49] R. J. Bell and A. E. Kingston, Proc. Phys. Soc. 88, 901 (1966).

[50] K. T. Tang, J. Chem. Phys. 55, 1064 (1971).

[51] A. Derevianko, W. R. Johnson, M. S. Safronova, and J. F. Babb, Phys. Rev. Lett. 82, 3589 (1999).

[52] M. S. Safronova, W. R. Johnson, and A. Derevianko, Phys. Rev. A 60, 4476 (1999).

[53] A. Derevianko, J. F. Babb, and A. Dalgarno, Phys. Rev. A 63, 052704 (2001).

[54] S. G. Porsev and A. Derevianko, J. Chem. Phys. 119, 844 (2003).

[55] J. Mitroy and M. W. J. Bromley, Phys. Rev. A 71, 019902(E),019903(E) (2005). 